\title{
Biofabrication of gold and silver nanoparticles for pharmaceutical applications
}

\author{
Hamed Barabadi ${ }^{1}$, Soheila Honary ${ }^{1}$ * \\ ${ }^{1}$ Department of Pharmaceutics, Faculty of Pharmacy Mazandaran University of Medical Sciences, Sari, Iran \\ Received: Nov 22, 2015, Revised: Dec 30, 2015, Accepted: Jan 23, 2016
}

\begin{abstract}
Biofabrication by using fungi is an exciting recent interest to develop an eco-friendly production of metallic nanoparticles (NPs) for pharmaceutical applications. This study aimed to synthesize and characterize gold ( $\mathrm{Au}$ ) and silver (Ag) NPs by using Penicillium simplisimum. The fungus was grown in fluid czapek dox broth on shaker at $28{ }^{\circ} \mathrm{C}$ and $200 \mathrm{rpm}$ for ten days. Then the supernatant was separated from the mycelia to convert $\mathrm{HAuCl}_{4}$ and $\mathrm{AgNO}_{3}$ solution into $\mathrm{Au}$ and $\mathrm{Ag}$ NPs separately. After 24 hours, synthesized $\mathrm{Au}$ and Ag NPs were characterized by using UV-Visible Spectroscopy as well as Photon Correlation spectroscopy (PCS) involves Polydispersity Index (PDI) and zeta potential. The UV-Visible Spectroscopy analysis revealed a plasmon bond peak around $533 \mathrm{~nm}$ and $400 \mathrm{~nm}$ suggesting formation of $\mathrm{Au}$ and Ag NPs, respectively. Furthermore, the PCS analysis showed an average diameter of $68 \mathrm{~nm}$ and $76 \mathrm{~nm}$ with PDI value of 0.2 and 0.23 for Au and Ag NPs, successively, which demonstrated that the nanoparticles formed with fairly well-defined dimensions and good monodispersity. Besides, a negative zeta potential were found for nanoparticles indicating their stability in the solution. The current approach suggests that the rapid synthesis of nanoparticles would be suitable for developing a green process for mass scale production. Besides, we believe that development of ecofriendly process for the formulation of metallic NPs is an important step in the field of application of nanotechnology and its optimization may make it a potential procedure for industrial production of NPs.
\end{abstract}

Keywords: Gold nanoparticles, silver nanoparticles, biofabrication, particle size, Penicillium simplisimum

Pharm Biomed Res 2016; 2(1): 1-7 ～DOI: 10.18869/acadpub.pbr.2.1.1

\section{Introduction}

Nanotechnology is an important field of modern pharmaceutical research involves synthesis and development of various nanoparticles (NPs) with unique features and extensive pharmaceutical and medical applications in drug delivery, diagnostics, imaging, sensing, gene delivery, artificial implants and tissue engineering (1). Silver (Ag) NPs are currently used as an active drug in targeted drug delivery (2), gene delivery (3), and artificial implants (4) and as a diagnostic agent for imaging and sensing in different diseases at their early stages. Owing to their mutationresistant antimicrobial activity, they are 
being used in different pharmaceutical formulations as antibacterial clothing (5), burn ointments (6) and coating for medical devices (7). Besides, gold ( $\mathrm{Au})$ compound and AuNPs have potential therapeutic applications include antiHIV activity, anti-angiogenesis $(8,9)$, anti-malarial agent (10) and antiarthritic agent (11). Fabrication of NPs could be undertaken using either chemical or biological systems (12). Biological methods as a more efficient and less hazardous way, synthesis metal nanoparticles and produces greater amounts of particles in less time and under milder conditions while using less hazardous reagents than the traditional preparation. Nowadays, the importance of biological synthesis is being emphasized globally because chemical methods are capital intensive, toxic, non-ecofriendly and have low productivity (13). Besides, it is important to understand the biosynthetic mechanism involved in the fabrication of metal NPs mediated by a biological system in order to gain better control of the process and products. Several microorganisms such as bacteria, fungi and yeasts have come up as nanofactories for synthesizing $\mathrm{Au}$ and AgNPs $(12,14,15)$. However, using of fungi in order to fabrication of NPs has drawn attention of workers because of its rapid, economical, eco-friendly protocol and it provides a single step technique for the biosynthesis process (16).The progress toward greener nanosynthesis has already been studied by different authors (12,14-18). Honary, et al. (2012-2015) applied different Penicillium species for extracellular synthesis of copper oxide, iron oxide, $\mathrm{Au}$ and Ag NPs $(12,14-18)$. The genus Penicillium seems to be extremely good candidates for the fabrication of $\mathrm{Au}$ and
Ag NPs. Penicillium is as a very common biomass waste from pharmaceutical industry, possesses a potential to fabricate nanoparticles and it would enhance the opportunity for cost-effective preparation of various $\mathrm{Au}$ and Ag NPs. In spite of all that, biological synthesis is still on the laboratory scale. Efforts should be made to investigate the practical application of microorganism in synthesis of metal nanoparticles. We believe that development of eco-friendly process for the synthesis of metallic nanoparticles is an important step in the field of application of nanotechnology. Therefore, the present study aimed to biofabricate $\mathrm{Au}$ and Ag NPs by using P. simplisimum.

\section{Materials and methods}

\section{Materials}

Yeast extract was purchased from Liofilchem, Italy. $\mathrm{HAuCl}_{4}, \mathrm{AgNO}_{3}$ and other chemical reagents were purchased from Merck Co., Germany. The pure colony of $P$. simplisimum was isolated from soil and approved by department of mycology and plant pathology of Sari Agriculture and Natural Resources University, Iran.

Preparation, purification and characterization of Au and AgNPs

The fungus $P$. simplisimum was cultured on fluid czapex dox broth including $21 \mathrm{~g}$ sucrose and $3 \mathrm{~g}$ yeast extract in $1000 \mathrm{~mL}$ distilled water and incubated on a rotary shaker at $200 \mathrm{rpm}$ for ten days at $28{ }^{\circ} \mathrm{C}$. The culture was centrifuged at $10,000 \mathrm{rpm}$ for $5 \mathrm{~min}$. Then the supernatant was separated from the mycelia to convert $\mathrm{HAuCl}_{4}$ and $\mathrm{AgNO}_{3}$ solutions into $\mathrm{Au}$ and $\mathrm{Ag}$ NPs separately. For that, $100 \mathrm{~mL}$ of $3 \mathrm{mM}$ solutions of $\mathrm{HAuCl}_{4}$ and $\mathrm{AgNO}_{3}$ 
were added to $100 \mathrm{~mL}$ of supernatant and incubated again for 24 hours at $28{ }^{\circ} \mathrm{C}$. In this step, the synthesized $\mathrm{Au}$ and $\mathrm{Ag}$ NPs were centrifuged at $20,000 \mathrm{rpm}$ by ultracentrifuge for 5 minutes to separate NPs from the solutions. Finally, the NPs redispersed in double distilled water and centrifuged again. This action was carried out for three times to separate purified NPs. Photon Correlation Spectroscopy (PCS), Polydispersity Index (PDI) and zeta potential of NPs were carried out to determine the size, distribution and stability of nanoparticles by using a Zetasizer Nanoparticle Analyzer 3600 at $25^{\circ} \mathrm{C}$ with a scattering angle of $90{ }^{\circ} \mathrm{C}$ (Malvern instruments, UK).

\section{Results}

Addition of $P$. simplisimum supernatant to $3 \mathrm{mM}$ aqueous $\mathrm{AgNO}_{3}$ solution led to the appearance of yellowish brown in solution after $24 \mathrm{~h}$ of reaction, indicating the formation of $\mathrm{Ag}$ NPs. Besides, addition of supernatant to $3 \mathrm{mM}$ aqueous $\mathrm{HAuCl}_{4}$ solution led to the appearance of dark purple in solution after $24 \mathrm{~h}$ of reaction, indicating the formation of $\mathrm{Au}$ NPs. Moreover, the UV-Vis spectrum exhibited an absorption band at around $533 \mathrm{~nm}$ and $400 \mathrm{~nm}$ which is a typical plasmon band suggesting formation of $\mathrm{Au}$ and $\mathrm{Ag}$ NPs respectively (Fig. 1 and 2). Moreover, the UV-vis spectrum revealed that low wavelength region recorded from the reaction medium exhibited an absorption band around $240 \mathrm{~nm}$ and it was attributed to aromatic amino acids of proteins. Furthermore, the PCS analysis showed an average diameter of $68 \mathrm{~nm}$ and $76 \mathrm{~nm}$ with PDI value of
0.2 And 0.23 for $\mathrm{Au}$ and $\mathrm{Ag} \mathrm{NPs}$, respectively, which demonstrated that the nanoparticles formed with fairly well-defined dimensions and good monodispersity (Fig. 3 and 4).

Besides, the zeta potentials of $\mathrm{Au}$ and $\mathrm{Ag}$ NPs were found at $-24 \mathrm{mV}$ and $-19 \mathrm{mV}$ which provides the repulsive force as an electrostatic stabilization (Fig. 5 and 6).

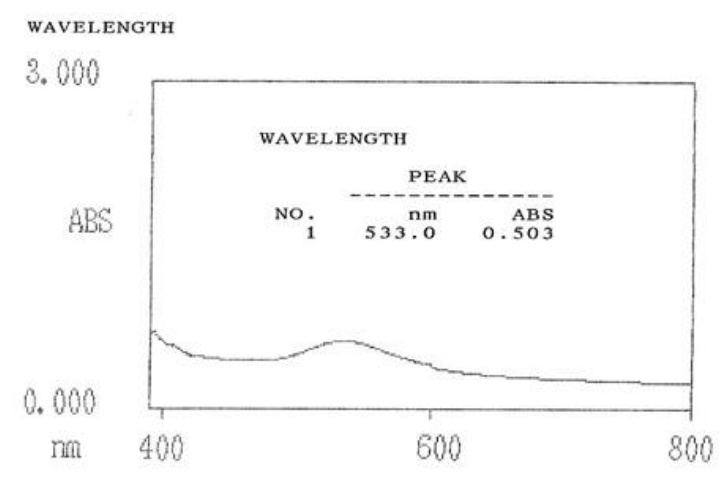

Figure $1 \mathrm{UV}-$ Visible absorption spectra of the $\mathrm{Au}$ NPs produced using Penicillium simplisimum.

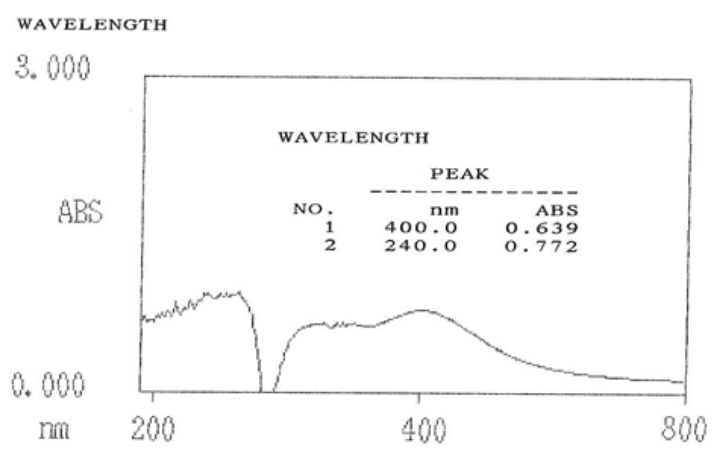

Figure 2 UV-Visible absorption spectra of the Ag NPs produced using Penicillium simplisimum. 


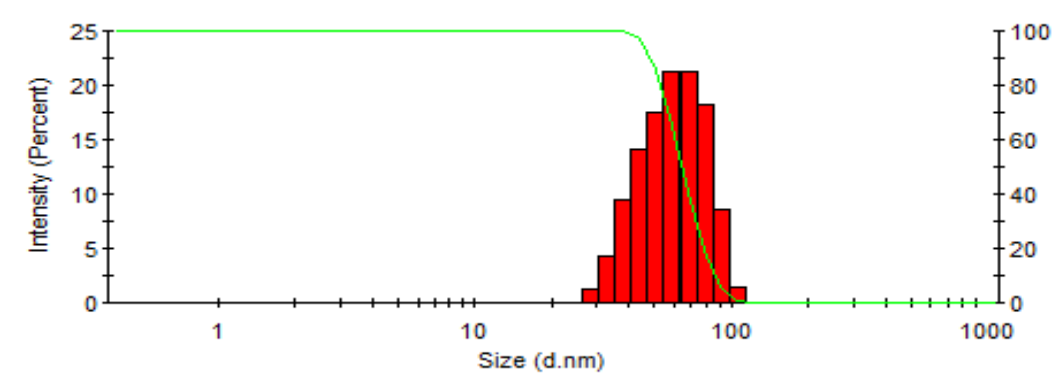

Figure 3 Photon Correlation Spectroscopy (PCS) of the Au NPs with the average size of 68 nm, PDI: 0.2 .

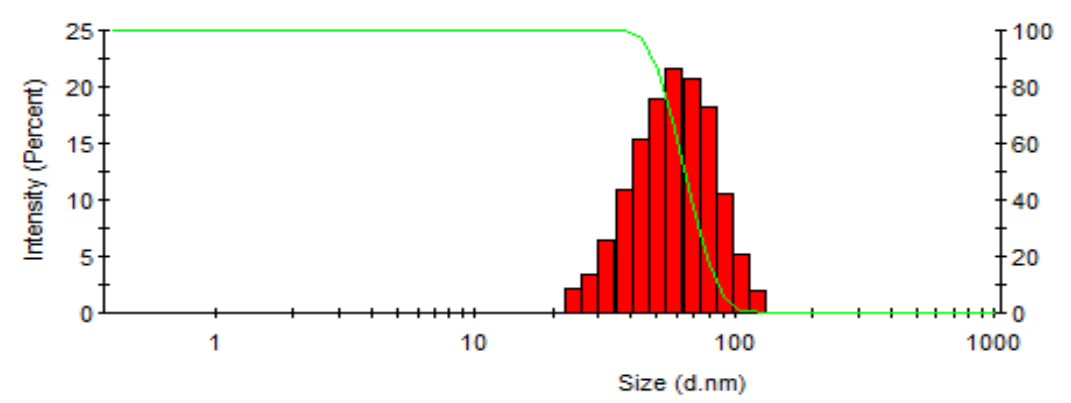

Figure 4 Photon Correlation Spectroscopy (PCS) of the Ag NPs with the average size of $76 \mathrm{~nm}$, PDI: 0.23 .

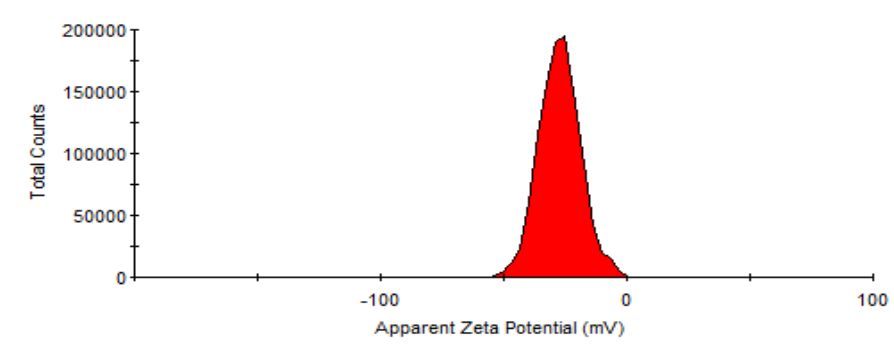

Figure 5 Zeta potential of the Au NPs was found at $-24 m V$.

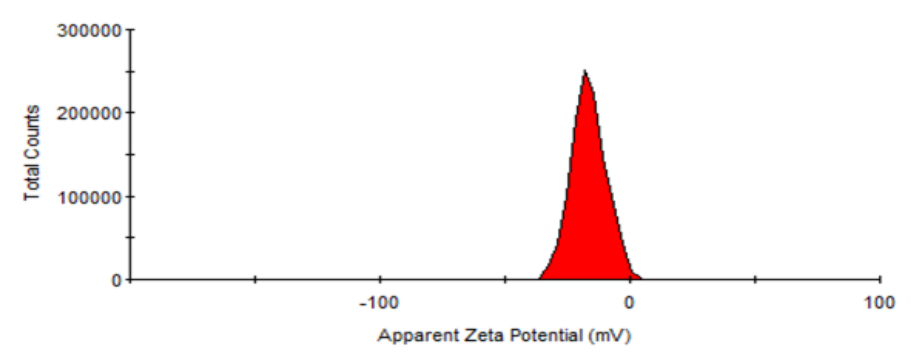

Figure 6 Zeta potential of the Ag NPs was found at $-19 \mathrm{mV}$. 


\section{Discussion}

The bioreduction of $\mathrm{Au}$ and $\mathrm{Ag}$ ions occurred in the supernatant and proteins might have critical role in formation and stabilization of the synthesized NPs. The color change from pale yellow to yellowish brown and dark purple when $3 \mathrm{mM}$ aqueous $\mathrm{AgNO}_{3}$ and $3 \mathrm{mM}$ aqueous $\mathrm{HAuCl}_{4}$ added respectively to the solutions, arises due to excitation of surface plasmon vibrations in the $\mathrm{Au}$ and $\mathrm{Ag}$ NPs $(12,14)$. Control without $\mathrm{Au}$ or $\mathrm{Ag}$ ions showed no change in color when incubated under same conditions. Many metals could be treated as free-electron systems. Such metal called plasma contains equal numbers of positive ions which are fixed in position and conduction electrons which are free and highly mobile. Under the irradiation of an electromagnetic wave, the free electrons are driven by the electric field to oscillate coherently. These collective oscillations of the free electrons are called plasmons. These plasmons can interact, under certain conditions, with visible lights in a phenomenon called surface plasmon resonance (SPR) (1519). Furthermore, this visible change in color due to SPR was accurately studied with UV-Vis absorption spectrophotometer analysis of colloidal $\mathrm{Au}$ and $\mathrm{Ag}$ solution. Figure 1 and 2 showed that the UV-Vis spectrum exhibited an absorption band at around $533 \mathrm{~nm}$ and $400 \mathrm{~nm}$ which is a typical plasmon band, suggesting formation of $\mathrm{Au}$ and $\mathrm{Ag}$ NPs respectively (14-19). The UV-Vis spectrum reveals that low wavelength region recorded from the reaction medium exhibited an absorption band around $240 \mathrm{~nm}$ and it was attributed to aromatic amino acids of proteins (Fig. 2). It is well known that the absorption band around $240 \mathrm{~nm}$ arises due to electronic excitation in tryptophan and tyrosine residue in the protein. This is in accordance with the results of previous studies carried out by this author (14-18). The biological agents secrete a large amount of enzymes, which are capable of hydrolyzing metals and thus bring about enzymatic reduction of metals ions (12). In case of fungi, the enzyme nitrate reductase is found to be responsible for the synthesis of nanoparticles $(12,14)$. The diagram of PCS analysis demonstrated that the NPs formed with fairly well-defined dimensions and good monodispersity (Fig. 3 and 4). Photon Correlation Spectroscopy facilitates the understanding of the dispersion and aggregation. The polydispersity index measures the second moment of the size distribution of the nanoparticle population. The PDI ranges are from a value of 0.01 for monodispersed particles and up to values of 0.5-0.7. Samples with very broad size distribution have polydispersity index values > 0.7 (14). The zeta potential of the nanoparticles provides the repulsive force as an electrostatic stabilization. The zeta potential of higher than $+30 \mathrm{mV}$ or lower than $-30 \mathrm{mV}$ depicts that the dispersion is stable and the zeta potential of zero means that the dispersion is unstable. Besides, the zeta potential strongly depends on the $\mathrm{pH}$ and the electrolyte concentration of the dispersed particles (20). Figure 5 and 6 showed that the zeta potential values of synthesized $\mathrm{Au}$ and $\mathrm{Ag}$ NPs were less negative than $-30 \mathrm{mV}$ maybe suggest that the electrostatic repulsive forces play a minor role for stabilization of these dispersions. Totally, the stability of particle dispersions depends on both steric stabilization and the balance between attractive and repulsive forces 
between the particles (DLVO theory) which is called electrostatic stabilization (21). It is suggested that secreted proteins and enzymes from fungi in the medium create a complex with nanoparticles and provide the steric stabilization, where a stabilizer is added to the dispersion adsorbing onto the particle surfaces and preventing them from coming close to one another in chemical methods (22).

\section{Conclusion}

The present study showed biofabricatation of Au and Ag NPs with fairly well-defined dimensions and good monodispersity by employing $P$. simplisimum. Synthesis and characterization of NPs is presently an important area of research, as selection of size and shape of NPs provides an efficient control over many of the physical and chemical properties. By using $P$. simplisimum for biofabrication of $\mathrm{Au}$ and $\mathrm{Ag} \mathrm{NPs}$, advantages of a natural nontoxic bioprocess could be highlighted because this method is considered as an eco-friendly contrary to the classic $\mathrm{Au}$ and Ag NPs synthesis

\section{References}

1. Morones JR, Elechiguerra JL, Camacho A, Holt K, Kouri JB. Ramfrez JT, Yacaman MJ. The bactericidal effect of silver nanoparticles. Nanotechnol 2005;16:2346-53.

2. Jain J, Arora S, Rajwade JM, Omray P, Khandelwal S, Paknikar KM. Silver nanoparticles in therapeutics: development of an antimicrobial gel formulation for topical use. Mol Pharm 2009;6:1388-401.

3. Roy K, Mao HQ, Huang SK. Leong KW. Oral gene delivery with chitosan-DNA nanoparticles generates immunologic protection in amurine model of peanut allergy. Nat Med 1999;5:387-91.

4. Sachlos E, Gotora D, Czernuszka JT. Collagen scaffolds reinforced with biomimetic composite nano-sized carbonate-substituted hydroxyapatite crystals and shaped by rapid prototyping to contain internal microchannels. Tissue Eng 2006;12:2479-87. methods that require expensive and toxic reducing, stabilizing, and functionalizing chemicals. Moreover, biofabrication not only synthesize NPs with unique shapes, but also achieve control over particle size seems to be a viable and convenient option. Furthermore, the extracellular synthesis of NPs could be highly valuable from the viewpoint of large-scale operations and easy downstream processing.

\section{Acknowledgment}

This work was financially supported by Traditional Medicine and Medica Research Center, Shaheed Beheshti University of Medical Sciences, Tehran, Iran.

\section{Conflict of interest statement}

The authors declared no potential conflict of interest with respect to the authorship, and/or publication of this study.

5. Vigneshwaran N, Kathe AA, Varadarajan PV, Nachane RP, Balasubramanya RH. Functional finishing of cotton fabrics using silver nanoparticles. J Nanosci Nanotechnol 2007;7:1893-7.

6. Ip M, Lui SL, Poon VKM, Lung I, Burd A. Antimicrobial activities of silver dressings: an in vitro comparison. J Med Microbiol 2006;55:5963.

7. Furno F, Morley KS, Wong B, Sharp BL, Arnold PL, Howdle SM, et al. Silver nanoparticles and polymeric medical devices: a new approach to prevention of infection? J Antimicrob Chemother 2004; 54:1019-24.

8. Yamaguchi $\mathrm{K}$, Ushijima $\mathrm{H}$, Hisano $\mathrm{M}$, Inoue $\mathrm{Y}$, Shimamura T, Hirano T, Muller WE. Immunomodulatory effect of gold sodium thiomalate on murine acquired immunodeficiency syndrome. Microbiol Immunol 2001;45:549-55. 
9. Mukherjee P, Bhattacharya R, Wang P, Wang L, Basu S, Nagy JA, Atala A, Mukhopadhyay D, Soker S. Antiangiogenic properties of gold nanoparticles. Clin Cancer Res 2005;11:3530-4.

10. Navarro M, Pérez H, Sanchez-Delgado R.A. Toward a novel metal-based chemotherapy against tropical diseases. 3. Synthesis and antimalarial activity in vitro and in vivo of the new gold-chloroquine complex [Au (PPh3)(CQ)] PF6. J Med Chem 1997;40:1937-9.

11. Tsai CY, Shiau AL, Chen SY, Chen YH, Cheng PC, Chang MY, Chen DH, Chou CH, Wang CR, $\mathrm{Wu}$ CL. Amelioration of collagen-induced arthritis in rats by nanogold. Arthritis Rheum 2007;56:544-54.

12. Honary S, Gharaei-Fathabad E, Barabadi $\mathrm{H}$. Fungus-mediated synthesis of gold nanoparticles: A novel biological approach to nanoparticle synthesis. J Nanosci Nanotechnol 2013;13:142730.

13. Kowshik M, Deshmukh N, Vogel W, Urban J, Kulkarni SK, Paknikar KM. Microbial synthesis of semiconductor CdS nanoparticles, their characterization, and their use in the fabrication of an ideal diode. Biotechnol Bioeng 2002;78:583-8.

14. Honary S, Barabadi H, Gharaei-Fathabad E, Naghibi F. Green synthesis of silver nanoparticles induced by the fungus Penicillium citrinum. Trop J Pharm Res 2013;12:7-11.

15. Barabadi H, Honary S, Ebrahimi P, Ali Mohammadi M, Alizadeh A, Naghibi F. Microbial mediated preparation, characterization and optimization of gold nanoparticles. Braz $\mathbf{J}$ Microb 2014;45:1493-501.
16. Honary S, Barabadi H, Gharaei-Fathabad E, Naghibi F. Green synthesis of copper oxide nanoparticles using Penicillium aurantiogriseum, Penicillium citrinum and Penicillium waksmanii. Dig J Nanomater Biostruct 2012;7:999-1005.

17. Honary S, Barabadi H, Ebrahimi P, Naghibi F, Alizadeh A. Development and optimization of biometal nanoparticles by using mathematical methodology: A microbial approach. J Nano R 2015;30:106-15.

18. Rahimi MT, Ahmadpour E, Rahimi EB, Spotin A, Kohansal Koshki MH, Alizadeh A, et al. Scolicidal activity of biosynthesized silver nanoparticles against Echinococcus granulosus protoscolices. Int J Surg 2015;19:128-33.

19. Soltani Nejad M, Shahidi Bonjar Gh, Khaleghi N. Biosynthesis of gold nanoparticles using Streptomyces fulvissimus isolate. Nanomed $\mathbf{J}$ 2015;2:153-9.

20. Bihari P, Vippola M, Schultes S, Praetner M, Khandoga AG, Reichel CA, Coester C, Tuomi T, Rehberg M, Krombach F. Optimized dispersion of nanoparticles for biological in vitro and in vivo studies. Part Fibre Toxicol 2008;5:14

21. Fritz G, Schadler V, Willenbacher N, Wagner NJ. Electrosteric Stabilization of Colloidal Dispersions. Langmuir 2002;18:6381-90.

22. Gericke M, Pinches A. Microbial production of gold nanoparticles. Gold Bull 2006;39:22-8. 